\title{
Two Numerical Methods to Solve the Second Order Multi-pantograph Equation with Boundary Conditions
}

\author{
Mahdi Ahmadinia*, Zeinab Safari \\ Department Mathematics, University of Qom, Qom, Iran \\ *Corresponding author: mahdiahmadinia72@gmail.com \& m-ahmadinia@qom.ac.ir
}

Received April 21, 2014; Revised May 28, 2014; Accepted June 18, 2014

\begin{abstract}
In this article, we present two numerical methods to solve the second order multi-pantograph equation with boundary conditions. The multi-pantograph equation is converted to an integral equation then the integral equation is solved by two projective methods. Some properties of Chebyshev polynomials are employed to prove the convergence analysis of the two proposed methods. Finally, numerical examples also are given to illustrate the efficiency and validity of the two proposed methods.
\end{abstract}

Keywords: Multi-pantograph equation, Integral equation, Chebyshev polynomials

Cite This Article: Mahdi Ahmadinia, and Zeinab Safari, "Two Numerical Methods to Solve the Second Order Multi-pantograph Equation with Boundary Conditions." Applied Mathematics and Physics, vol. 2, no. 4 (2014): 124-127. doi: 10.12691/amp-2-4-1.

\section{Introduction}

The following second order delay differential

$$
\left\{\begin{array}{l}
y^{\prime \prime}(t)+p(t) y^{\prime}(t)+\sum_{i=1}^{m} g_{i}(t) y\left(q_{i} t\right)=f(t), \quad t \in[0, b] \\
y(0)+y(b)=0, \\
y^{\prime}(0)+y^{\prime}(b)=0,
\end{array}\right.
$$

is called multi-pantograph equation with anti-periodic boundary conditions when $0<q_{i}<1, \quad i=1, \ldots, m-1$, $q_{m}=1$ and $m \in \mathbb{N}$. The first order of the multi-pantograph equation has been solved by many methods such as Bessel collection method [12] by Yüzbaşi et al. the Taylor approximation method $[6,7,8]$ by Sezer et al. and $\theta$ method [4] by Liu and Li. Saadatmandi and Dehghan applied the variational iteration method in [5] and $\mathrm{Yu}$ in [15] as well. Homotopy method has been employed in [10] by Yusufoğlu. Brunner at al. [2] applied discontinuous Galerkin method to solve the delay differential equations of pantograph type and explained convergence analysis of the method completely. Some other cases of the neutral delay differential equation have been studied in $[13,14]$ by Yüzbaşi et al. The system of multi-pantograph equation of the first order has been solved by Bessel collocation method in [11]. The present paper introduces two projective methods to solve the second order of multipantograph equation with anti periodic boundary conditions. We convert multi-pantograph equation (1) to an integral equation and solve it by two projective methods. The rest of the paper is arranged as fallows:
Section 2 introduces preliminaries of the method and describes the method which is to convert problem (1) to an integral equation also the integral equation is solved by two projective methods. Section 3 proves the convergence analysis of the two projective methods. The last section illustrates numerical examples to confirm the theory.

\section{Preliminaries and the Method}

Multi-pantograph equation (1) with anti-periodic boundary conditions can be converted to the following integral equation, for convenience assume that $m=3$ and $0<q_{1}<q_{2}<q_{3}=1$. The value $m$ is fixed throughout the paper, $m=3$. Consider

$$
u(t)=f(t)+\int_{0}^{b} K(s, t) u(s) d s,
$$

when

$$
\left\{\begin{array}{l}
K(s, t)=Z(s, t)+H(s, t), \\
H(s, t)=\frac{p(t)}{2}+\sum_{i=1}^{3} \frac{g_{i}(t)}{4}\left(-b+2 s-2 q_{i} t\right),
\end{array}\right.
$$

and

$$
Z(s, t)= \begin{cases}\sum_{i=1}^{3} g_{i}(t)\left(q_{i} t-s\right), & 0<s<q_{1} t \\ \sum_{i=2}^{3} g_{i}(t)\left(q_{i} t-s\right), & q_{1} t<s<q_{2} t \\ t-s, & q_{2} t<s<t \\ -p(t), & t<s<b .\end{cases}
$$


If $u(t)$ is the solution of integral equation (2) then the following formula yields the solution of multi-pantograph equation(1).

$$
y(t)=\frac{b+2 t}{-4} \int_{0}^{b} u(s) d s+\frac{1}{2} \int_{0}^{b} s u(s) d s+\int_{0}^{b}(t-s) u(s) d s .
$$

To obtain the solution of multi-pantograph equation (1) with anti-periodic boundary conditions, it is sufficient to solve integral equation (2) and considering (3). Therefore, we solve integral equation (2) by two projective methods and obtain the approximate solution of (1) by approximate solution (2). Integral equation (2) can be converted to the same integral equation on $[-1,1]$ by changing variable, then, without loss of generality, assume that $[0, b]=[-1,1]$. To present the two projective methods, we have to introduce Chebyshev polynomials. Let $T_{j}$ denotes Chebyshev polynomial of degree $j$ as follows:

$$
\begin{aligned}
& T_{j}(t)=\cos (j \arccos (t)), \quad-1 \leq t \leq 1, \\
& t_{i}=\cos \left(\frac{(2 i-1) \pi}{2 j}\right), \quad i=1, \ldots, j .
\end{aligned}
$$

Note that $t_{i}$ 's are the zeros of $T_{j}(t)$. Let $V_{n}$ be the polynomial space of degree $n$. Consider the two projections as follows:

$$
\left\{\begin{array}{l}
\prod_{n}^{G}: C[-1,1] \rightarrow V_{n}, \\
\prod_{n}^{G} f(t)=\sum_{i=0}^{n} \frac{2}{\pi}\left\langle f, T_{i}\right\rangle T_{i}(t), \\
\prod_{n}^{I}: C[-1,1] \rightarrow V_{n} \\
\prod_{n}^{I} f(t)=\sum_{i=0}^{n} f\left(t_{i}\right) l_{i}(t)
\end{array}\right.
$$

when $t_{i}$ 's are the zeros of $T_{n}$ introduced in (4) and $l_{i}$ is Lagrange polynomial

$$
l_{i}(x)=\prod_{j=0, j \neq i}^{n} \frac{\left(x-t_{j}\right)}{\left(t_{i}-t_{j}\right)}, \quad 0 \leq i \leq n .
$$

Conside $u_{n}(t):=\sum_{i=0}^{n} c_{i} T_{i}(t)$ as an approximation solution of (2) in the finite dimensional space $V_{n}$. We will obtain the unknown coefficients $c_{i}, \quad 0 \leq i \leq n$, by two projective methods. Integral equation (2) yields

$$
\sum_{i=0}^{n} c_{i} T_{i}(t)=f(t)+\int_{0}^{b} K(t, s) \sum_{i=0}^{n} c_{i} T_{i}(s) d s .
$$

Taking projection $\prod_{n}^{G}$ on both sides of (5) implies

$$
\begin{aligned}
& \prod_{n}^{G}\left(\sum_{i=0}^{n} c_{i} T_{i}(t)\right) \\
& =\prod_{n}^{G}(f(t))+\prod_{n}^{G}\left(\int_{0}^{b} K(t, s) \sum_{i=0}^{n} c_{i} T_{i}(s) d s\right) .
\end{aligned}
$$

Both sides of the above equation are two polynomials of degree $n$, this equality of polynomials yields a system of $(n+1)$ equations with $(n+1)$ unknowns $c_{i}, i=0, \ldots, n$ then we can obtain unknowns $c_{i}, i=0, \ldots, n$ by solving a linear system. This method is called Galerkin method. We have another linear system with unknowns $c_{i}, i=0, \ldots, n$ if we apply the projection $\prod_{n}^{I}$ instead of $\prod_{n}^{G}$ in (6). This projective method is called collocation method. Both projective methods solve integral equation (2) approximately and the approximate solution converges to the exact solution of (2). By considering (3) and the approximate solution of (2), we will obtain the approximate solution of (1), which converges to the exact solution (1). We will prove this fact in the next section.

\section{Convergence Analysis}

This section proves that the approximate solutions of two projective methods converge to the exact solution of integral equation (2). This fact will be presented by theorem 3.1 and corollary 3.2. The approximate solution of the integral equation yields an approximate solution of multi-pantograph equation with anti-periodic boundary conditions (1) which converges to the exact solution of (1), it is the result of theorem 3.3.

Theorem 3.1. Assume that $\mathcal{K}: V \rightarrow V$, is bounded operator

$$
\mathcal{K} u(t)=f(t)+\lambda \int_{a}^{b} K(s, t) u(s) d s,
$$

and assume $\lambda-\mathcal{K}: V \rightarrow V$ is one to one and onto. Further assume $\left\|\mathcal{K}-P_{n} \mathcal{K}\right\| \rightarrow 0$ as $n \rightarrow \infty$, where $P_{n}$ is a projection $P_{n}: V \rightarrow V_{n}$ and $V_{n}$ is a finite dimensional space. Then for all sufficiently large $n$, say $n \geq N$, the operator $\left(\lambda-P_{n} \mathcal{K}\right)^{-1}$ exists as a bounded operator. Moreover, it is uniformly bounded:

$$
\sup _{n \geq N}\left\|\left(\lambda-P_{n} \mathcal{K}\right)^{-1}\right\|<\infty .
$$

For the approximate solution $u_{n}$ and $u$ of

$$
\left(\lambda-P_{n} \mathcal{K}\right) u_{n}=P_{n} f, u_{n} \in V,
$$

and $(\lambda-\mathcal{K}) u=f$ respectively, we have

$$
\frac{|\lambda|}{\left\|\lambda-P_{n} \mathcal{K}\right\|}\left\|u-P_{n} u\right\| \leq\left\|u-u_{n}\right\| \leq|\lambda|\left\|\left(\lambda-P_{n} \mathcal{K}\right)^{-1}\right\|\left\|u-P_{n} u\right\| .
$$

Proof: This theorem has been presented by Atkinson and Han [1] page 479.

Corollary 3.2. Assume that $[a, b]=[-1,1], V=C^{2}[-1,1]$ and $V_{n}$ is the polynomial space of degree $n$. If $u_{n}$ is the approximate solution of (7) and $u$ is the exact solution of (2), then $u_{n}$ converges to $u$ in the two following cases:

i) $P_{n}=\prod_{n}^{G}$,

ii) $P_{n}=\prod_{n}^{I}$,

where $\prod_{n}^{G}$ and $\prod_{n}^{I}$ introduced in the previous section.

Proof: Theorem 3.1 yields

$$
\left\|u_{n}-u\right\| \leq M\left\|P_{n} u-u\right\|,
$$

it is sufficient to show that $\left\|P_{n} u-u\right\|$ converges zero in two cases $P_{n}=\prod_{n}^{I}$ and $P_{n}=\prod_{n}^{G}$. Trefethen proved 
$\left\|\Pi_{n}^{G} u-u\right\|$ converges zero in the recent paper [9]. It is obvious that $\prod_{n}^{I}$ is interpolation projection at zeros of Chebyshev polynomials, then, $\left\|\Pi_{n}^{I} u-u\right\|$ converges to zero when the sequence $\left\|u^{(n)}\right\|$ is uniformly bounded (See Burden and Faires [3] page 524).

Theorem 3.3. Let $u$ be the solution of integral equation (2) then the function $y$ in (3) is a solution of the multipantograph equation with anti-periodic boundary conditions (1). Moreover, if $u_{n}$ is an approximate solution of integral equation (2) and $y_{n}$ is approximate solution (1) which is obtained by substituting $u_{n}$ in (3), then the approximate solution $y_{n}$ converges to the exact solution (1), when

$$
\left\|u_{n}-u\right\| \rightarrow 0, n \rightarrow \infty .
$$

Proof. Let $u$ be the exact solution of integral equation (2). It is clear that the defined function $y$ in (3) satisfies the multi-pantograph equation with anti-periodic boundary conditions (1). If $y_{n}$ is an approximate solution (1), which is obtained by (3) and $u_{n}$ (approximate solution (2)) then the following inequality is clear by considering (3),

$$
\left\|y_{n}-y\right\| \leq C\left\|u_{n}-u\right\|,
$$

where $C$ is a constant independent of $n$. The above inequality proves that the approximate solution $y_{n}$ converges to the exact solution of the multi-pantograph equation with anti-periodic boundary conditions.

Let $u_{n}=\sum_{i=0}^{n} c_{i} T_{i}$ be the solution of the following system:

$$
u_{n}(t)-P_{n} \mathcal{K} u_{n}(t)=P_{n} f(t),
$$

where $P_{n}=\prod_{n}^{I} \quad$ or $P_{n}=\prod_{n}^{G}$, then corollary 3.2. and theorem 3.3. imply that $y_{n}$ converges to the exact solution of multi-pantograph equation (1).

\section{Numerical Examples}

This section confirms the theory of the two proposed methods by illustrating numerical examples. The tables show the error of the methods $\left(\left\|y_{n}-y\right\|_{\infty}\right)$ and CPU time. All computations of the following examples have been run by Maple 15.Software.

Example 4.1. Consider the following multi-pantograph equation with anti-periodic boundary conditions,

$$
\left\{\begin{array}{l}
y^{\prime \prime}(t)+\sin (t) y^{\prime}(t)+\sum_{i=0}^{3} g_{i}(t) y\left(q_{i} t\right)=f(t), \quad t \in[0, \pi], \\
y(0)+y(\pi)=0, \\
y^{\prime}(0)+y^{\prime}(\pi)=0,
\end{array}\right.
$$

where

$$
\begin{cases}g_{1}(t)=\exp (t), & q_{1}=0.5 \\ g_{2}(t)=t^{2}, & q_{2}=0.75 \\ g_{3}(t)=\frac{1}{4} \cos \left(\frac{2 t}{5}\right), & q_{3}=1,\end{cases}
$$

and

$$
\begin{aligned}
f(t)= & 1 / 32\left(-8 \cos (t)+4 t^{2}-4 \pi t\right) \cos (2 t / 5) \\
& -t^{2} \cos (3 t / 4)-\exp (t) \cos (t / 2) \\
& -\cos ^{2}(t)+\cos (t)+t / 8(t-2 \pi) \exp (t) \\
& +(t-\pi / 2) \sin (t)+9 t^{4} / 4+3 \pi t^{3} / 8+2
\end{aligned}
$$

The exact solution is $y(t)=-\cos (t)+\frac{t^{2}}{2}-\frac{-\pi t}{2}$. Table 1 presents the error of the methods and CPU time. Figures 1. and figure 2. illustrate the error of Galerkin method and collocation method respectively.

Table 1. Error and CPU time

\begin{tabular}{|c|c|c|c|c|}
\hline $\mathrm{N}$ & $\begin{array}{c}\text { Collocation } \\
\text { error }\end{array}$ & $\begin{array}{c}\text { Collocation } \\
\text { CPU time }\end{array}$ & $\begin{array}{c}\text { Galerkin } \\
\text { error }\end{array}$ & $\begin{array}{c}\text { Galerkin } \\
\text { CPU time }\end{array}$ \\
\hline 4 & $4.90 \mathrm{e}-02$ & 0.125 & $2.86 \mathrm{e}-02$ & 5.491 \\
\hline 8 & $3.89 \mathrm{e}-06$ & 0.109 & $2.59 \mathrm{e}-06$ & 28.751 \\
\hline 16 & $5.47 \mathrm{e}-16$ & 0.672 & $4.50 \mathrm{e}-16$ & 317.571 \\
\hline 32 & $3.63 \mathrm{e}-40$ & 3.642 & $1.46 \mathrm{e}-36$ & 7161.235 \\
\hline 64 & $1.53 \mathrm{e}-97$ & 35.32 & - & - \\
\hline 128 & $5.03 \mathrm{e}-231$ & 305.29 & - & - \\
\hline
\end{tabular}

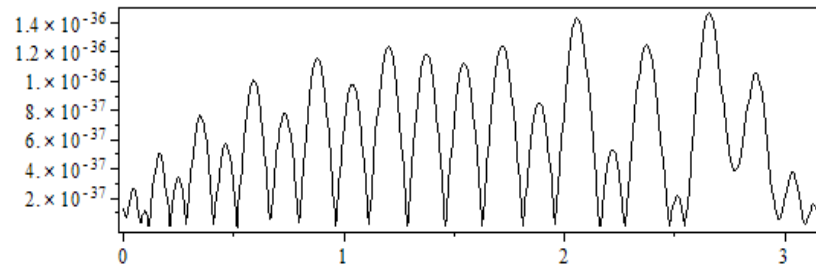

Figure 1. Ex.4.1. Galerkin method $\mathrm{N}=32$

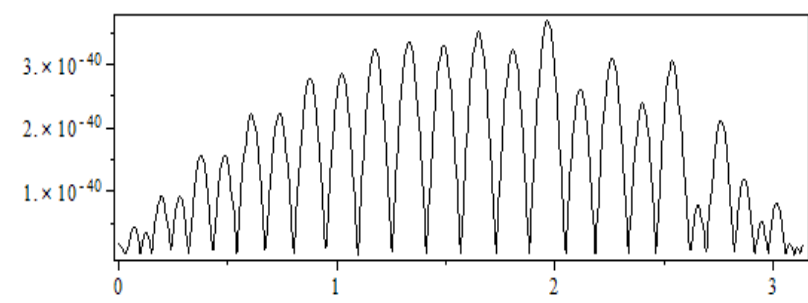

Figure 2. Ex.4.1. Collocation method $\mathrm{N}=32$

Example 4.2. Consider the following multi-pantograph equation with anti-periodic boundary conditions,

$$
\left\{\begin{array}{l}
y^{\prime \prime}(t)+\sin (2 t) y^{\prime}(t)+\sum_{i=0}^{3} g_{i}(t) y\left(q_{i} t\right)=f(t), \quad t \in[0, \pi] \\
y(0)+y(\pi)=0 \\
y^{\prime}(0)+y^{\prime}(\pi)=0 .
\end{array}\right.
$$

where 


$$
\begin{cases}g_{1}(t)=0.25+0.5 t^{2}, & q_{1}=0.15, \\ g_{2}(t)=\exp (t), & q_{2}=0.45, \\ g_{3}(t)=t^{2}, & q_{3}=1 .\end{cases}
$$

and

$$
\begin{aligned}
& f(t)=((\cos (t)+\sin (t)) \exp (t)+7.04 t) \sin (2 t) \\
& -26.06 t^{2}+2.70+\exp (t) \sin (0.45 t) \exp (0.45 t) \\
& +\left(0.25+0.5 t^{2}\right) \sin (0.15 t) \exp (0.15 t)+ \\
& \left(2 \cos (t)+t^{2} \sin (t)-17.38+0.71 t^{2}\right) \exp (t)+3.56 t^{4}
\end{aligned}
$$

The exact solution of the multi-pantograph with boundary condition is $y(t)=\sin (t) \exp (t)$

$$
+(\pi / 4-t / 2 \pi)(1-\exp (\pi)) \text {. }
$$

Table 2 shows the error of the methods and CPU time. Figures.3 and figures. 4 show the error of Galerkin method and collocation method respectively.

Table 2. Error and CPU time

\begin{tabular}{|c|c|c|c|c|}
\hline $\mathrm{N}$ & $\begin{array}{c}\text { Collocation } \\
\text { error }\end{array}$ & $\begin{array}{c}\text { Collocation } \\
\text { CPU time }\end{array}$ & $\begin{array}{c}\text { Galerkin } \\
\text { error }\end{array}$ & $\begin{array}{c}\text { Galerkin } \\
\text { CPU time }\end{array}$ \\
\hline 4 & $4.90 \mathrm{e}-01$ & 0.078 & $2.95 \mathrm{e}-01$ & 3.61 \\
\hline 8 & $7.20 \mathrm{e}-04$ & 0.107 & $3.86 \mathrm{e}-04$ & 15.437 \\
\hline 16 & $1.36 \mathrm{e}-12$ & 0.547 & $8.49 \mathrm{e}-13$ & 137.406 \\
\hline 32 & $2.29 \mathrm{e}-34$ & 3.485 & $1.24 \mathrm{e}-34$ & 393.594 \\
\hline 64 & $3.20 \mathrm{e}-87$ & 31.730 & - & - \\
\hline 128 & $4.50 \mathrm{e}-211$ & 307.141 & - & - \\
\hline
\end{tabular}

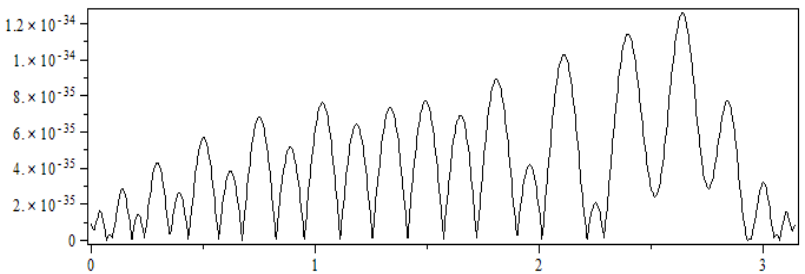

Figure 3. Ex. 4.2. Galerkin method $\mathrm{N}=32$

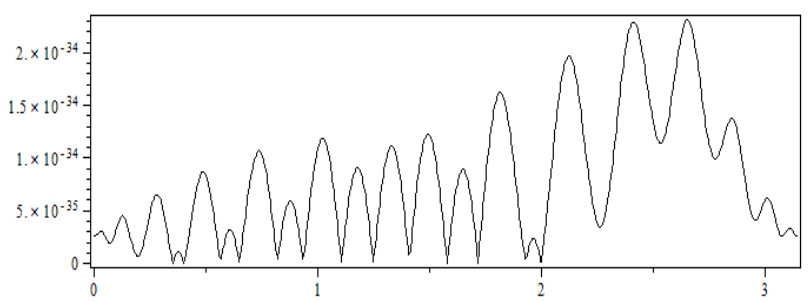

Figure 4. Ex. 4.2. Collocation method $N=32$

Figure 5 and Figure 6 illustrate the error of the collocation method for Example 4.1-4.2 and $N=128$.

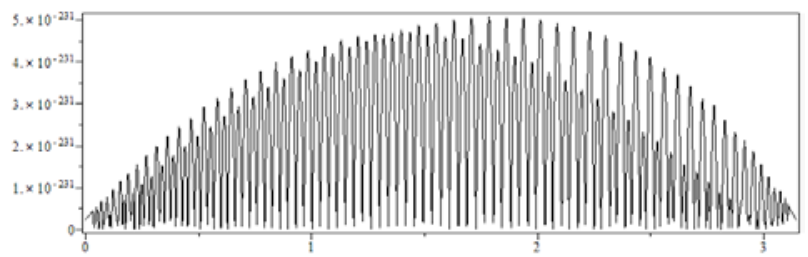

Figure 5. Ex.4.1. Collocation method $\mathrm{N}=128$

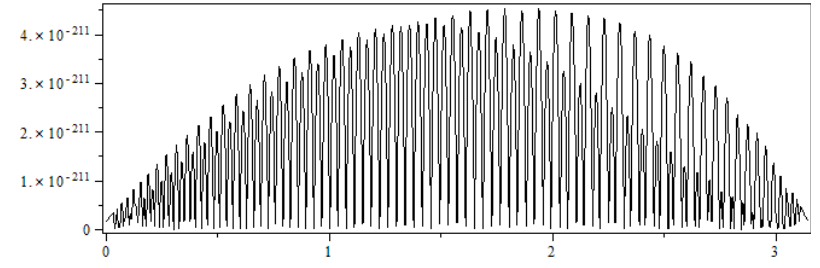

Figure 6. Ex.4.2. Collocation method $\mathrm{N}=128$

\section{Conclusion}

In this study, we convert the second order multipantograph equation with anti-periodic boundary conditions to an integral equation then two projective methods are proposed to solve the integral equation. Some properties of interpolation and Chebyshev polynomials prove the convergence analysis of the two proposed methods. The numerical examples show that the errors of the two methods are same approximately, but the collocation method spends CPU time less than Galerkin method.

\section{References}

[1] K. Atkinson and W. Han, Theoretical Numerical Analysis A Functional Analysis Frame-work, Springer-Vergla, New York, 2009.

[2] H. Brunner, Q. Huang and H. Xie, "Discontinuous Galerkin methods for delay differential equations of pantograph type," SIAM J. Numer. Anal, 48. 1944-1967. 2010.

[3] R. L. Burden and J. D. Faires, Numerical Analysis, Brooks/Cole, Boston, 2011.

[4] M.Z. Liu and D. Li, "Properties of analytic solution and numerical solution of multi-pantograph equation,”Appl. Math. Comput., 155. 853-871. 2004.

[5] A. Saadatmand and M. Dehghan, "Variational iteration method for solving a generalized pantograph equation,” Comput. Math. Appl. 58(11-12). 2190-2196. 2009.

[6] M. Sezer and A. Akyüz-Daşcioğlu, "A Taylor method for numerical solution of generalized pantograph equations with linear functional argument,”J. Comput. Appl. Math., 200. 217-225. 2007.

[7] M. Sezer, S. Yalçinbaş and M. Gülsu,“A Taylor polynomial approach for solving generalized pantograph equations with nonhomogeneous term,” Int. J. Comput. Math., 85(7), 1055-1063. 2008.

[8] M. Sezer, S. Yalçinbaş and N. Şahin, "Approximate solution of multi-pantograph equation with variable coefficients," J. Comput. Appl. Math., 214. 406-416. 2008.

[9] L. N. Trefethen, "Is Gauss quadrature better than ClenshawCurtis?,” SIAM Review, 50. 67-87. 2008.

[10] E.Yusufoğlu, "An efficient algorithm for solving generalized pantograph equations with linear function alargument," Appl. Math. Comput, 217(7). 3591-3595. 2010.

[11] Ş. Yüzbaşi, "An efficient algorithm for solving multi-pantograph equations systems,” Comput. Math. Appl, 64, 589-603, 2012.

[12] S.Yüzbaşi, N. Şahin, and M. Sezer. "A Bessel collocation method for numerical solution of generalized pantograph equations." Numerical Methods for Partial Differential Equations, 28(4). 1105-1123, 2012.

[13] Ş.Yüzbaşi, N. Şahin and M. Sezer, "Numerical solutions of systems of linear Fredholm integro-differential equations with Bessel polynomial bases,” Comput. Math. Appl, 61(10), 30793096, 2011.

[14] Ş.Yüzbaşi, N. Şahin and M. Sezer, “A Bessel polynomial approach for solving linear neutral delay differential equations with variable coefficients,” J. Adv. Res. Differ. Equ. 3(1). 81-101, 2011.

[15] Z. H. Yu, "Variational iteration method for solving the multipantograph delay equation,” Phys. Lett. A, 372(43). 6475-6479. 2008. 